\title{
Differential Diagnosis of Intraplaque Hemorrhage and Dissection on High-Resolution MR Imaging in Patients with Focal High Signal of the Vertebrobasilar Artery on TOF Imaging
}

\author{
Jung Hwan Kim, Hyo Sung Kwak* ${ }^{\mathbb{D}}$, Seung Bae Hwang $\mathbb{D}^{-}$and Gyung Ho Chung
}

check for updates

Citation: Kim, J.H.; Kwak, H.S.; Hwang, S.B.; Chung, G.H. Differential Diagnosis of Intraplaque Hemorrhage and Dissection on High-Resolution MR Imaging in Patients with Focal High Signal of the Vertebrobasilar Artery on TOF Imaging. Diagnostics 2021, 11, 1024. https://doi.org/10.3390/

diagnostics11061024

Academic Editor: Andrea D. Annoni

Received: 21 May 2021

Accepted: 2 June 2021

Published: 3 June 2021

Publisher's Note: MDPI stays neutral with regard to jurisdictional claims in published maps and institutional affiliations.

Copyright: (C) 2021 by the authors Licensee MDPI, Basel, Switzerland. This article is an open access article distributed under the terms and conditions of the Creative Commons Attribution (CC BY) license (https:// creativecommons.org/licenses/by/ $4.0 /)$.
Department of Radiology and Research Institute of Clinical Medicine of Jeonbuk National University, Biomedical Research Institute of Jeonbuk National University Hospital, Jeonju 54896, Korea; hanbobo@hanmail.net (J.H.K.); sbh1010@jbnu.ac.kr (S.B.H.); chunggh@jbnu.ac.kr (G.H.C.)

* Correspondence: kwak8140@jbnu.ac.kr; Tel.: +82-63-250-2582; Fax: +82-63-272-0481
Abstract: Purpose: Intraplaque hemorrhage (IPH) and dissection in the vertebrobasilar artery (VBA) on time of flight (TOF) source imaging are seen as focal eccentric high-signal intensity. The purpose of this study is to identify IPH and dissection in the VBA using high-resolution magnetic resonance imaging (HR-MRI). Methods: A total of 78 patients (VBA IPH: 55; dissection: 23) with focal highsignal intensity in the VBA on simultaneous non-contrast angiography and intraplaque hemorrhage (SNAP) of HR-MRI were included in this study. The focal high-signal intensity in the VBA on SNAP was defined as $>200 \%$ than that of the adjacent muscle. We analyzed the signal intensity ratio (area of focal high signal intensity area/lumen) on TOF imaging and black blood (BB) T2-weighted imaging. Results: The VBA IPH group was older than the dissection group and had more hypertension. Signal intensity of a false lumen in patients with dissection on TOF imaging was significantly higher than that of VBA IPH $(p<0.001)$. The signal intensity ratio between lumen and lesion on TOF imaging was significantly higher in the dissection group $(p<0.001)$. The signal intensity of a false lumen in patients with dissection on BB T2-weighted imaging was significantly lower than that of VBA IPH $(p<0.001)$. The signal intensity ratio between lumen and lesion on BB T2-weighted imaging was significantly higher in the VBA IPH group $(p<0.001)$. Conclusions: TOF imaging and BB T2-weighted imaging on HR-MRI in patients with focal eccentric high-signal intensity on TOF imaging can distinguish between VBA IPH and dissection.

Keywords: intracranial artery; atherosclerosis; MRI; vessel wall imaging; dissection

\section{Introduction}

The posterior cerebral circulation, also known as the vertebrobasilar system, consists of the basilar and vertebral arteries and contributes about $20 \%$ of the brain's blood supply [1]. Posterior circulation ischemic stroke accounts for only about $20-30 \%$ of all ischemic stroke, but it has higher mortality and morbidity rates compared with anterior circulation ischemic stroke $[2,3]$. It is known that the major cause of vertebrobasilar artery (VBA) stenosis is atherosclerosis with or without intraplaque hemorrhage (IPH) [4]. However, vertebrobasilar artery dissection accounts for some VBA stenosis.

IPH is thought to be caused by the rupture of fragile and tortuous neovessels formed within the plaque [5]. IPH is considered to be an important atherogenic stimulus that advances macrophage infiltration, which makes the plaque more unstable [6,7]. Dissection is a rare cause of stroke; however, it represents one of the more common causes of stroke in patients younger than 45 years of age $[8,9]$. Especially, it frequently occurs in the carotid artery or vertebral artery with high mobility. Dissection was not easy to diagnose in the past, but the diagnosis is becoming easier as vascular imaging technology has developed [10-12].

$\mathrm{IPH}$ has a different etiology from dissection, and it requires different treatment strategies to treat patients with suspected VBA stenosis. However, in general, atherosclerotic 
plaque or dissection is observed as simple stenosis in time-of-flight (TOF) MR angiography (MRA) [13,14]. On the other hand, high-resolution magnetic resonance imaging (HR-MRI) is very useful for showing various blood vessel conditions. Therefore, it is expected that changes caused by various etiologies, such as atherosclerosis or dissection, can be distinguished on HR-MRI [15]. IPH and dissection in the VBA on TOF imaging is seen as focal eccentric high-signal intensity [15]. The differences in signal intensity between IPH and dissection in the VBA on HR-VWI are not fully known. The aim of this study was to distinguish between VBA IPH and dissection by signal intensity on TOF imaging and black blood (BB) T2-weighted imaging on HR-VWI.

\section{Materials and Methods}

\subsection{Patients}

This study was approved by the local institutional review board, and informed consent was obtained from all patients before imaging. Between January 2015 and December 2019, we consecutively selected patients for VBA stenosis related to atherosclerosis or suspicious dissection using TOF-MRA. During this period, all patients underwent standard brain MRI and MRA to detect any other lesions. We performed HR-MRI for evaluation of VBA lesions within 1 week after the initial MR examination. Patients with any of the following features were excluded from the analysis: (1) Massive and dominant calcified plaque; (2) complete BA occlusion on MRA and HR-MRI; (3) other vasculopathies, such as inflammatory arteritis or Moyamoya disease; (4) dissecting aneurysm; (5) clinical contraindications to MRI; (6) no IPH in the VBA atherosclerosis; or (7) insufficient MR imaging quality to evaluate contrast enhancement of the aneurysmal wall.

\subsection{High-Resolution $M R$ Imaging}

MRI was performed with a 3T MRI scanner (Achieva; Philips Medical Systems, Amsterdam, The Netherlands) with a 16-channel head coil. All patients initially underwent conventional brain MRI, which included 3D TOF-MRA. TOF-MRA of the axial plane was obtained for each patient, and data were reconstructed using a dedicated online postprocessing tool to determine blood vessel architecture.

The HR-MRI protocol included 5 MR scans: BB T1-weighted, BB T2-weighted, TOF axial, simultaneous non-contrast angiography and intraplaque hemorrhage (SNAP), and contrast-enhanced BB T1-weighted imaging. BB T1-weighted imaging was acquired using a 2D turbo spin-echo sequence with the following imaging parameters: repetition time $/$ echo time $=800 / 10 \mathrm{~ms}$, field of view $=140 \times 140 \mathrm{~mm}$, matrix size $=140 \times 150$, slice thickness $=2.0 \mathrm{~mm}$, echo train length $=10$, and number of excitations $=2$. Gadodiamide (0.1 mmol/kg body weight; Dotarem; Guerbet, Aulnay-sous-Bois, France) for contrast-enhanced BB T1-weighted imaging was injected as a bolus intravenously in all patients. Contrast-enhanced BB T1-weighted imaging was carried out $\sim 5$ min after contrast injection. SNAP sequence was performed for evaluation of optimal IPH as hyperintense. Image parameters were as follows: $\mathrm{TR} / \mathrm{TE} / \mathrm{TI}=10 / 4.7 / 490 \mathrm{~ms}, \mathrm{FA}=11^{\circ}, \mathrm{ETL}=98$, FOV $=149 \times 149 \mathrm{~mm}$, matrix $=187 \times 216$. The total scan time was 25 to $30 \mathrm{~min}$, and patients remained in the MR machine for 35 to $45 \mathrm{~min}$.

\subsection{Clinical Data Assessment}

Patients were classified as having either a symptomatic or asymptomatic lesion according to the presence of recent ischemic stroke. Symptomatic stenosis was defined as a diffusion-restrictive lesion seen on DWI in the territory of the stenotic VBA with a corresponding acute neurologic deficit within 2 weeks before MR imaging. Clinical data, including basic demographics and risk factors for atherosclerosis, namely diabetes, hypertension, dyslipidemia, current smoking, and history of coronary disease, were also recorded. 


\subsection{Image Analysis}

All MR images were reviewed retrospectively by 2 neuroradiologists (with 25 years and 15 years of experience, respectively) blinded to the clinical information of each patient. They assessed image quality by consensus using a 4-scale scoring system (1, poor; 2 , adequate; 3 , good; 4 , excellent). Images with a score of 1 were excluded from the final analysis. Disagreements regarding image quality were resolved by consensus.

VBA Plaque was defined as a thickening of the focal wall relative to image slices from beneath or above the focal wall, as identified on BB T2- and T1-weighted imaging. VBA IPH was defined as a signal intensity greater than $150 \%$ of T1 SNAP signal of adjacent muscle [16]. VBA dissection was defined as a wall thickening with low-signal intensity because of an intimal flap on BB T2-weighted imaging and high-signal intensity because of an intimal flap on TOF-MRA $[17,18]$. We analyzed the presence of VBA IPH and dissection among all samples using HR-MRI. Two neuroradiologists performed all these procedures. Consensus interpretation was used for the final analysis when the readers' interpretations differed.

The percentage of stenosis was estimated on TOF-MRA using the formula of $[(a-b) / a]$ $\times 100 \%(a=$ narrowed vessel diameter, $\mathrm{b}=$ proximal normal vessel diameter). Because of the small diameters of intracranial vessels, we focused on stenoses involving the larger, more proximal intracranial arteries. In addition, maximal wall thickness was measured at the highest point of plaque on T1-weighted imaging.

We measured the signal intensity of the VBA plaque and vessel lumen on TOF source imaging and BB T2-weighted imaging. The signal intensity ratio between VBA plaques and vessel lumen on BB T2-weighted imaging and TOF source imaging was measured at the stenotic area.

\subsection{Statistical Analysis}

The proportion of patients with VBA plaque with VBA IPH and dissection was investigated. The demographics and risk factors were compared to these patients. Pearson's chi-square test and Student's $t$-test were used for categorical and continuous variables as appropriate. A 2 -sided $p$-value of $<0.05$ was considered statistically significant. Receiveroperating characteristic (ROC) curves of IPH or dissection were assessed to evaluate the diagnostic efficacy for detecting VBA IPH on BB T2-weighted imaging and TOF source imaging. Statistical analysis was performed by using SPSS 23.0 for Windows (SPSS, IBM, Chicago, IL, USA) and MedCalc software, version 16.4.2 (MedCalc, Ostend, Belgium).

\section{Results}

\subsection{Patients}

During the study period, 155 patients underwent HR-MRI for evaluation of VBA plaques or stenosis. Of these patients, 58 were excluded from this study due to no IPH in the VBA atherosclerosis, 7 because of poor quality, 6 because of dissecting aneurysms, and 7 because of massive calcification in the VBA plaques. In total, 78 patients (45 males; mean age, 67.9 years) with VBA IPH or dissection were enrolled. Of these patients, 55 had VBA IPH and 23 had VBA dissection.

\subsection{Comparison of Clinical Characteristics between IPH and Dissection Groups}

The results of the comparison between patients with VBA IPH and dissection are shown in Table 1. The mean age of patients with VBA dissection was significantly younger, while IPH patients with VBA plaque were the oldest $(56.6 \pm 3.1$ years old for VBA dissection, $72.7 \pm 1.2$ years old for VBA plaque with IPH; $p<0.001$ ). When the risk factors of atherosclerosis were compared, VBA plaque with IPH patients had the highest levels of hypertension $(85.5 \%$ vs. $47.8 \% ; p<0.001)$. Previous stroke history was the most prevalent in IPH groups $(25.2 \%$ vs. $4.3 \%$; $p=0.055)$. Symptomatic lesions were similar between the two groups. 
Table 1. Baseline characteristics between VBA IPH and VBA dissection.

\begin{tabular}{ccccc}
\hline & $\begin{array}{c}\text { All Patients } \\
(\boldsymbol{n}=\mathbf{7 8})\end{array}$ & $\begin{array}{c}\text { VBA Atherosclerotic Plaque } \\
(\boldsymbol{n}=\mathbf{5 5 )}\end{array}$ & $\begin{array}{c}\text { VBA Dissection } \\
(\boldsymbol{n}=\mathbf{2 3})\end{array}$ & $\boldsymbol{p}$-Value \\
\hline Age (years) & $67.9 \pm 1.5$ & $72.7 \pm 1.2$ & $56.6 \pm 3.1$ & $<0.001$ \\
Male, $\mathrm{n}(\%)$ & $45(57.7)$ & $31(56.4)$ & $14(60.9)$ & 0.713 \\
Hypertension, $\mathrm{n}(\%)$ & $58(74.4)$ & $47(85.5)$ & $11(47.8)$ & $<0.001$ \\
Diabetes mellitus, $\mathrm{n}(\%)$ & $23(29.5)$ & $18(32.7)$ & $5(21.7)$ & 0.332 \\
Hyperlipidemia, $\mathrm{n}(\%)$ & $25(32.1)$ & $19(34.5)$ & $6(26.1)$ & 0.465 \\
Alcohol drinking, n (\%) & $26(33.3)$ & $17(30.9)$ & $9(39.1)$ & 0.482 \\
Smoking, $\mathrm{n}(\%)$ & $23(29.5)$ & $14(25.5)$ & $9(39.1)$ & 0.227 \\
Previous stroke, $\mathrm{n}(\%)$ & $15(19.2)$ & $14(25.5)$ & $1(4.3)$ & 0.055 \\
Previous heart disease, $\mathrm{n}(\%)$ & $17(21.8)$ & $14(25.5)$ & $3(13.0)$ & 0.229 \\
Symptomatic, $\mathrm{n}(\%)$ & $32(41.0)$ & $21(38.2)$ & $11(47.8)$ & 0.430 \\
\hline
\end{tabular}

VBA, vertebrobasilar artery; IPH, intraplaque hemorrhage.

\subsection{Signal Intensity Ratio of BB T2-Weighted and TOF Imaging between IPH and Dissection Groups}

The single intensity ratios of VBA plaques on BB T2-weighted and TOF imaging between IPH and dissection groups are shown in Table 2. VBA dissection groups on TOF imaging had significantly brighter signal intensity compared to VBA IPH groups $(608.4 \pm 64.6$ vs. $326.6 \pm 24.4 ; p<0.001)$. In addition, the lumen-lesion signal intensity ratio on TOF imaging was significantly higher in VBA dissection groups $(80.7 \pm 6.8$ vs. $45.4 \pm 2.4 ; p<0.001)$. VBA IPH groups on BB T2-weighted imaging had significantly bright signal intensity compared to VBA dissection groups ( $540.9 \pm 53.5 \mathrm{vs.} 319.8 \pm 124.0$; $p<0.001$ ). In addition, the lesion-lumen signal intensity ratio on BB T2-weighted imaging was significantly higher in VBA IPH groups (1095.5 \pm 162.0 vs. $285.2 \pm 81.6 ; p<0.001)$. The maximal wall thickness and degree of stenosis were similar between the two groups.

Table 2. High-resolution MR imaging between VBA IPH and VBA dissection.

\begin{tabular}{cccc}
\hline & $\begin{array}{c}\text { VBA Atherosclerotic Plaque } \\
(\boldsymbol{n}=\mathbf{5 5})\end{array}$ & VBA Dissection $(\boldsymbol{n = 2 3 )}$ & $\boldsymbol{p}$-Value \\
\hline Maximal wall thickness, mm & $2.0 \pm 1.7$ & $2.0 \pm 1.1$ & 0.817 \\
Stenosis, \% & $48.5 \pm 2.28$ & $52.8 \pm 3.2$ & 0.297 \\
Lumen SI on TOF & $753.1 \pm 53.8$ & $750.9 \pm 63.2$ & 0.981 \\
Lesion SI on TOF & $326.6 \pm 24.4$ & $608.4 \pm 64.6$ & $<0.001$ \\
SI ratio on TOF & $45.4 \pm 2.4$ & $80.7 \pm 6.8$ & $<0.001$ \\
Lumen SI on BB T2 imaging & $113.0 \pm 15.8$ & $95.7 \pm 29.3$ & 0.525 \\
Lesion SI on BB T2 imaging & $540.9 \pm 53.5$ & $319.8 \pm 124.0$ & $<0.001$ \\
SI ratio on BB T2 imaging & $1095.5 \pm 162.0$ & $285.2 \pm 81.6$ & $<0.001$ \\
Wall enhancement, $\mathrm{n}(\%)$ & $5(5.5)$ & $3(13.0)$ & 0.353 \\
\hline
\end{tabular}

VBA, vertebrobasilar artery; IPH, intraplaque hemorrhage; TOF, time of flight; BB, black blood; SI, signal intensity.

\subsection{Comparison of ROC Analysis between BB T2-Weighted and TOF Imaging}

The ROC curve analysis of the signal intensity ratio between both groups is shown in Table 3. The ROC curve analysis revealed a higher diagnostic value for analysis between VBA IPH and dissection for both imaging modalities (TOF imaging: AUC $=0.852$, $95 \% \mathrm{CI}=0.760-0.943, p<0.001$; BB T2-weighted imaging: $\mathrm{AUC}=0.851,95 \% \mathrm{CI}=0.743-0.958$, $p<0.001$ ) (Figures 1 and 2). 
Table 3. ROC curve analysis of signal intensity ratio between both groups.

\begin{tabular}{ccccccccc}
\hline & AUC & $\mathbf{9 5} \% \mathbf{C I}$ & $\boldsymbol{p}$-Value & Sensitivity & Specificity & PPV & NPV & Cutoff \\
\hline $\begin{array}{c}\text { TOF: SI ratio: } \\
\text { T2: SI ratio: }\end{array}$ & 0.852 & $0.760-0.943$ & $<0.001$ & 0.739 & 0.945 & 1 & 0.775 & 217.4 \\
\begin{tabular}{c} 
Plaque/Lumen \\
\hline
\end{tabular} & 0.851 & $0.743-0.958$ & $<0.001$ & 0.826 & 0.727 & 0.333 & 0.917 & 53.4 \\
\hline
\end{tabular}

AUC: area under curve, PPV: positive predictive value, NPV: negative predictive value.

Intraplaque hemorrhage
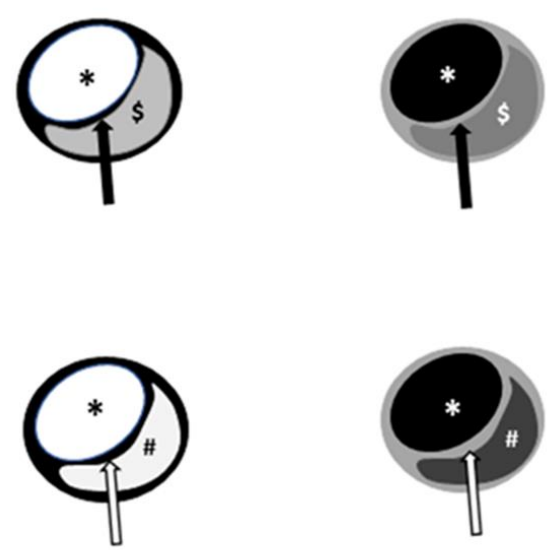

\section{Dissection}

Figure 1. Illustration of HR-VWI between intraplaque hemorrhage and dissection. Dissection shows a similar signal intensity between the true lumen and false lumen on time of flight and black blood T2weighted imaging. Note: black arrow, fibrous cap; white arrow, intimal flap; ${ }^{*}$, lumen, \$, intraplaque hemorrhage; \#, false lumen.

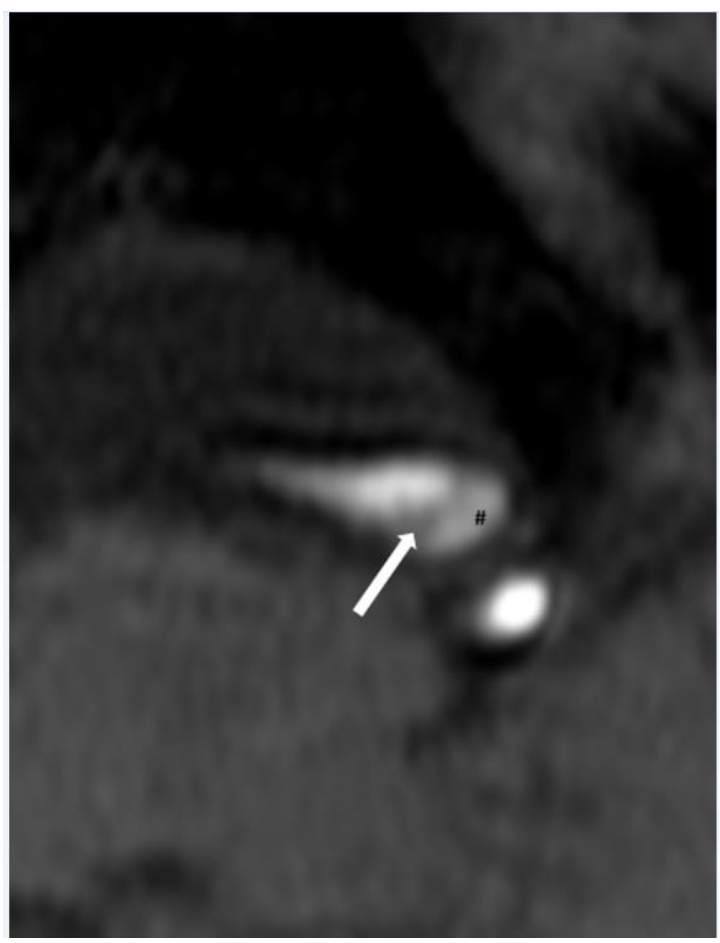

(A)

Figure 2. Cont. 


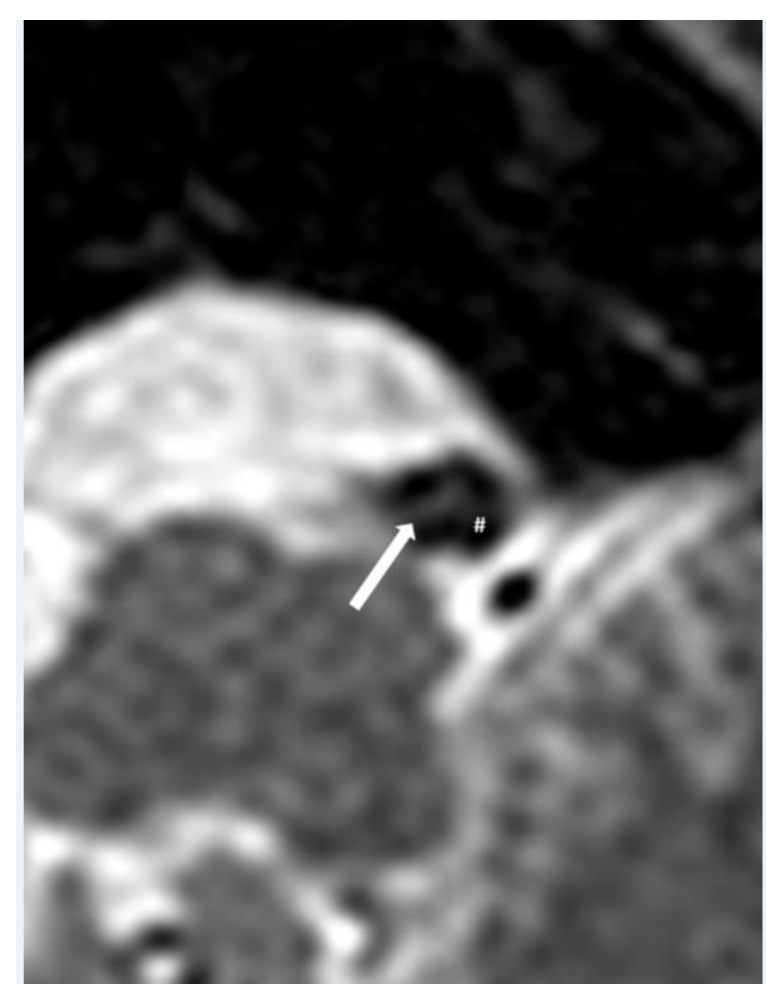

(B)

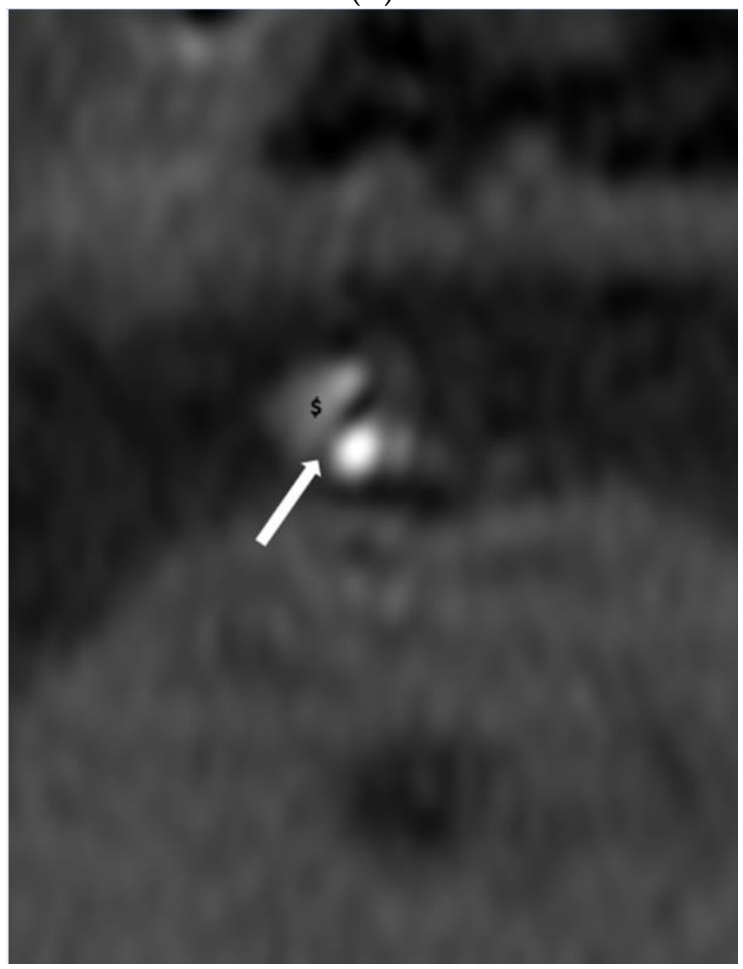

(C)

Figure 2. Cont. 


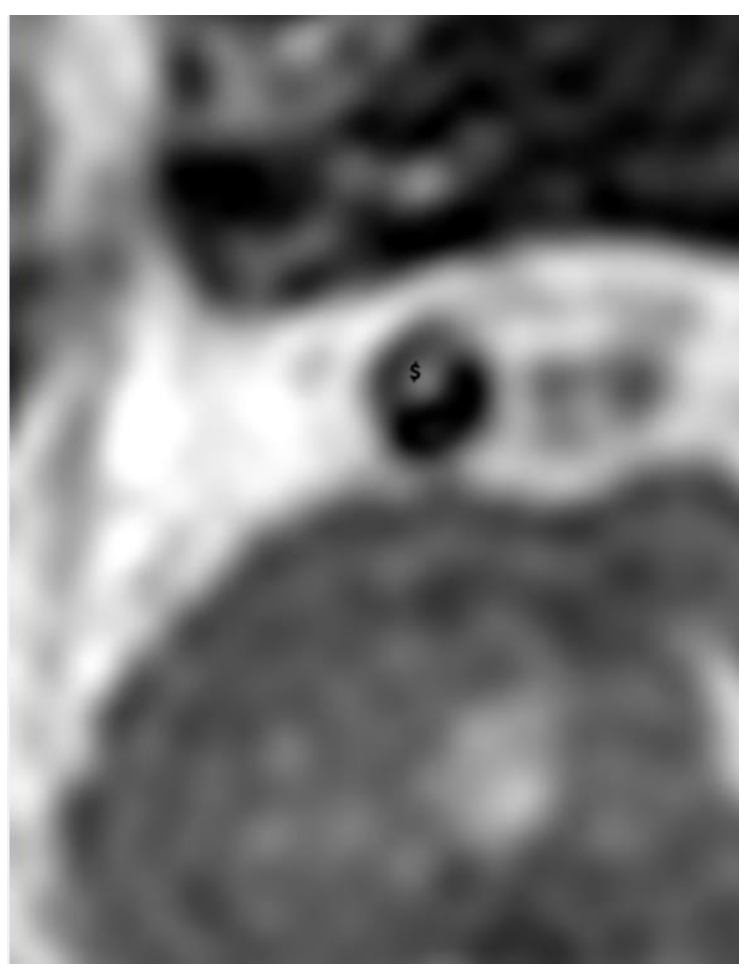

(D)

Figure 2. (A,B). Dissection. (A) Time-of-flight imaging shows a similar high signal intensity between the true lumen and false lumen (\#). Note the intimal flap with low signal intensity (white arrow). (B) Black blood T2-weighted imaging shows a similar low signal intensity of true and false lumen (\#) between the intimal flap (white arrow). (C,D). Intraplaque hemorrhage. (C) Intraplaque hemorrhage (\$) on time-of-flight imaging shows a relatively subtle high signal intensity compared to the high signal intensity of the lumen. Note the fibrous cap with low signal intensity (white arrow). (D) Plaque of basilar artery on black blood T2-weighted imaging shows the iso signal intensity compared to the low signal intensity of the lumen.

\section{Discussion}

In this study, VBA IPH showed a significantly lower signal intensity compared to a false lumen of dissection on TOF source imaging, and a false lumen of dissection showed a significantly lower signal intensity compared to VBA IPH on BB T2-weighted source imaging. VBA IPH and dissection can be differentiated on BB T2-weighted and TOF source imaging on HR-VWI.

Atherosclerotic plaque is the most important cause of stroke, and is commonly associated with unstable plaque. Especially, IPH is a common feature of atherosclerotic plaques and is an important feature of complex lesions preceding acute stroke events [19]. $\mathrm{IPH}$ presence plays a more important role in the progression of plaque during the long term $[20,21]$ and increases the risk of ipsilateral stroke events $[16,22]$. Therefore, early detection of atherosclerotic IPH is very useful for the management of atherosclerosis and the prevention of stroke.

Patients with intracranial dissection most often present with a nonspecific headache followed by ischemic stroke or subarachnoid hemorrhage (SAH). Dissecting aneurysms related to SAH and transmural dissection show a fusiform or irregular dilatation by extension toward the adventitia [23]. Sub-intimal dissection related to ischemic stroke shows a focal stenosis/occlusion or strings of pearls sign [23]. Thus, identifying dissecting aneurysms on imaging studies is easier for correct diagnosis and management. However, dissection presented by focal stenosis or complete occlusion is very difficult to distinguish between other stroke causes. In this study, as mentioned above, patients with a dissecting aneurysm were excluded from the analysis. In addition, in our study, young age was a 
factor significantly affecting VBA dissection. However, hypertension was a factor that significantly affected VBA IPH.

Detection of IPH commonly uses T1-weighted MR imaging techniques such as fast spin-echo imaging, TOF, MPRAGE imaging, or SNAP imagings. Ota et al. [24] reported that among T1-weighted images, MPRAGE sequences demonstrated superior diagnostic accuracy for detecting and quantifying carotid IPH compared to other T1 weighted imaging modalities and compared to histology. According to Li et al. [25,26], SNAP could detect carotid IPH with a smaller size and has higher sensitivity in detecting hemorrhage compared with MPRAGE in a clinical and histological study. We used SNAP imaging as a reference for the detection of VBA IPH.

The radiological features of arterial dissection are the intimal flap, double lumen, intramural hematoma, pseudoaneurysm, stenosis, and occlusion. However, because these were formed primarily by using luminal angiography, these criteria may not apply to HRMRI [27]. In a study by Wang et al., intramural hematoma (61\% of patients), double lumen (50\% of patients), and intimal flap ( $42 \%$ of patients) were recognized as the characteristic features of dissection by high-resolution MRI [28]. However, recent studies in patients with middle cerebral artery dissection reported that features of a double lumen and intimal flap are most common on HR-MRI [18,29-31]. When HR-MRI shows intramural hematoma without a double lumen or intimal flap, intracranial artery dissection should be considered first in the differential diagnosis. However, an interpreter may have to take into account IPH in equivocal cases that also demonstrate the clinical features of atherosclerosis. The differentiation between IPH and intramural hematoma may sometimes be difficult and may rely on clinical information such as patient age, atherosclerotic risk factors, symptoms, and change of radiologic findings on follow-up [15].

In this study, we investigated the signal intensity of IPH and dissection in patients with VBA stenosis on BB T2-weighted imaging and TOF imaging. The two categories sometimes showed vascular hyperintensity on T1-weighted imaging because of an IPH or intramural hematoma [32]. In addition, TOF source imaging showed a high signal intensity in an IPH or intramural hematoma. In our study, the signal intensity of a false lumen in the dissection group on TOF imaging showed a bright high signal intensity compared to the signal intensity of IPH. A false lumen of dissection on BB T2-weighted imaging showed a dark signal intensity by flow suppression of the BB technique compared to the signal intensity of plaque. These findings suggest that a false lumen of dissection might result in continuous or turbulent flow rather than a clot or thrombus due to flow suppression from the BB technique and similar signal intensity of a true lumen on TOF imaging and BB T2weighted imaging may be seen. The diagnostic performance of detection of VBA dissection using our criteria revealed a higher value for both imaging modalities in ROC analysis.

There are some limitations to this study. First, the 78 patients with 55 VBA IPH and 23 VBA dissection were retrospectively recruited. Future studies with larger samples are needed. Second, this retrospective study has a nonrandomized study design; the possibility exists that patients in this study may constitute a high-risk subgroup who require MR images of the head and neck. Finally, a final diagnosis of IPH and dissection was performed by trained neuroradiologists using HR-MRI without histological analysis. The diagnosis of intracranial vessel disease based on MR imaging without histological analysis is a problem that cannot be solved.

\section{Conclusions}

VBA IPH shows a relatively low signal intensity compared to a false lumen of dissection on TOF imaging, and a false lumen of dissection showed a relatively low signal intensity compared to VBA IPH on BB T2-weighted imaging on HR-MRI. TOF imaging and BB T2-weighted imaging on HR-MRI in patients with focal eccentric high-signal intensity on TOF imaging can distinguish between VBA IPH and dissection. 
Author Contributions: J.H.K. and H.S.K.; writing-original draft preparation, G.H.C. and S.B.H.; writing-review and editing, H.S.K.; funding acquisition All authors have read and agreed to the published version of the manuscript.

Funding: This research was supported by Basic Science Research Program through the National Research Foundation of Korea (NRF) funded by the Ministry of Education (NRF-2020R1A2C1008089).

Institutional Review Board Statement: This study was conducted according to the guidelines of the Declaration of Helsinki, and approved by the Institutional Review Board of Jeunbuk National Hospital (JUH 2019-10-040).

Informed Consent Statement: The requirement for patient informed consent was waived for review of patient records and images.

Conflicts of Interest: The authors declare that they have no conflict of interest.

\section{References}

1. Roth, E.J. Vertebrobasilar System. In Encyclopedia of Clinical Neuropsychology; Kreutzer, J.S., DeLuca, J., Caplan, B., Eds.; Springer: New York, NY, USA, 2011; pp. 2608-2609. [CrossRef]

2. Mehndiratta, M.; Pandey, S.; Nayak, R.; Alam, A. Posterior circulation ischemic stroke—clinical characteristics, risk factors, and subtypes in a north Indian population: A prospective study. Neurohospitalist 2012, 2, 46-50. [CrossRef]

3. Kubik, C.S.; Adams, R.D. Occlusion of the basilar artery-A clinical and pathological study. Brain 1946, 69, 73-121. [CrossRef]

4. Cloud, G.; Markus, H. Diagnosis and management of vertebral artery stenosis. QJM Int. J. Med. 2003, 96, 27-54. [CrossRef]

5. Virmani, R.; Kolodgie, F.D.; Burke, A.P.; Finn, A.V.; Gold, H.K.; Tulenko, T.N.; Wrenn, S.P.; Narula, J. Atherosclerotic plaque progression and vulnerability to rupture: Angiogenesis as a source of intraplaque hemorrhage. Arterioscler. Thromb. Vasc. Biol. 2005, 25, 2054-2061. [CrossRef] [PubMed]

6. Altaf, N.; Daniels, L.; Morgan, P.S.; Auer, D.; MacSweeney, S.T.; Moody, A.R.; Gladman, J.R. Detection of intraplaque hemorrhage by magnetic resonance imaging in symptomatic patients with mild to moderate carotid stenosis predicts recurrent neurological events. J. Vasc. Surg. 2008, 47, 337-342. [CrossRef] [PubMed]

7. Kolodgie, F.D.; Gold, H.K.; Burke, A.P.; Fowler, D.R.; Kruth, H.S.; Weber, D.K.; Farb, A.; Guerrero, L.; Hayase, M.; Kutys, R. Intraplaque hemorrhage and progression of coronary atheroma. N. Engl. J. Med. 2003, 349, 2316-2325. [CrossRef] [PubMed]

8. Zhou, Y.; Wang, L.; Zhang, J.-R.; Zhou, K.; Shuai, J.; Gong, Z.-L.; Liu, Y.; Wei, F.; Han, Y.-F.; Shen, L. Angioplasty and stenting for severe symptomatic atherosclerotic stenosis of intracranial vertebrobasilar artery. J. Clin. Neurosci. 2019, 63, 17-21. [CrossRef]

9. Markus, H.S.; Levi, C.; King, A.; Madigan, J.; Norris, J. Antiplatelet therapy vs anticoagulation therapy in cervical artery dissection: The Cervical Artery Dissection in Stroke Study (CADISS) randomized clinical trial final results. JAMA Neurol. 2019, 76, 657-664. [CrossRef]

10. Ma, N.; Lou, X.; Zhao, T.-Q.; Wong, E.-H.-C.; Jiang, W.-J. Intraobserver and interobserver variability for measuring the wall area of the basilar artery at the level of the trigeminal ganglion on high-resolution MR images. Am. J. Neuroradiol. 2011, 32, E29-E32. [CrossRef]

11. Huang, B.; Yang, W.-Q.; Liu, X.-T.; Liu, H.-J.; Li, P.-J.; Lu, H.-K. Basilar artery atherosclerotic plaques distribution in symptomatic patients: A 3.0 T high-resolution MRI study. Eur. J. Radiol. 2013, 82, e199-e203. [CrossRef]

12. Pasta, S.; Agnese, V.; Di Giuseppe, M.; Gentile, G.; Raffa, G.M.; Bellavia, D.; Pilato, M. In Vivo Strain Analysis of Dilated Ascending Thoracic Aorta by ECG-Gated CT Angiographic Imaging. Ann. Biomed. Eng. 2017, 45, 2911-2920. [CrossRef]

13. Mehdi, E.; Aralasmak, A.; Toprak, H.; Yildiz, S.; Kurtcan, S.; Kolukisa, M.; Asil, T.; Alkan, A. Craniocervical dissections: Radiologic findings, pitfalls, mimicking diseases: A pictorial review. Curr. Med. Imaging 2018, 14, 207-222. [CrossRef]

14. Kerwin, W.S.; Hatsukami, T.; Yuan, C.; Zhao, X.-Q. MRI of carotid atherosclerosis. Am. J. Roentgenol. 2013, 200, W304-W313. [CrossRef]

15. Choi, Y.J.; Jung, S.C.; Lee, D.H. Vessel wall imaging of the intracranial and cervical carotid arteries. J. Stroke 2015, 17, 238. [CrossRef]

16. Yu, J.H.; Kwak, H.S.; Chung, G.H.; Hwang, S.B.; Park, M.S.; Park, S.H. Association of Intraplaque Hemorrhage and Acute Infarction in Patients With Basilar Artery Plaque. Stroke 2015, 46, 2768-2772. [CrossRef] [PubMed]

17. Han, M.; Rim, N.J.; Lee, J.S.; Kim, S.Y.; Choi, J.W. Feasibility of high-resolution MR imaging for the diagnosis of intracranial vertebrobasilar artery dissection. Eur. Radiol. 2014, 24, 3017-3024. [CrossRef] [PubMed]

18. Kwak, H.S.; Hwang, S.B.; Chung, G.H.; Jeong, S.K. High-resolution magnetic resonance imaging of symptomatic middle cerebral artery dissection. J. Stroke Cerebrovasc. Dis. 2014, 23, 550-553. [CrossRef] [PubMed]

19. Levy, A.P.; Moreno, P.R. Intraplaque hemorrhage. Curr. Mol. Med. 2006, 6, 479-488. [CrossRef] [PubMed]

20. McNally, J.S.; McLaughlin, M.S.; Hinckley, P.J.; Treiman, S.M.; Stoddard, G.J.; Parker, D.L.; Treiman, G.S. Intraluminal thrombus, intraplaque hemorrhage, plaque thickness, and current smoking optimally predict carotid stroke. Stroke 2015, 46, 84-90. [CrossRef]

21. Sun, J.; Underhill, H.R.; Hippe, D.S.; Xue, Y.; Yuan, C.; Hatsukami, T.S. Sustained acceleration in carotid atherosclerotic plaque progression with intraplaque hemorrhage: A long-term time course study. JACC Cardiovasc. Imaging 2012, 5, 798-804. [CrossRef] 
22. Schindler, A.; Schinner, R.; Altaf, N.; Hosseini, A.A.; Simpson, R.J.; Esposito-Bauer, L.; Singh, N.; Kwee, R.M.; Kurosaki, Y.; Yamagata, S.; et al. Prediction of Stroke Risk by Detection of Hemorrhage in Carotid Plaques: Meta-Analysis of Individual Patient Data. JACC Cardiovasc. Imaging 2020, 13, 395-406. [CrossRef]

23. Debette, S.; Compter, A.; Labeyrie, M.A.; Uyttenboogaart, M.; Metso, T.M.; Majersik, J.J.; Goeggel-Simonetti, B.; Engelter, S.T.; Pezzini, A.; Bijlenga, P.; et al. Epidemiology, pathophysiology, diagnosis, and management of intracranial artery dissection. Lancet Neurol. 2015, 14, 640-654. [CrossRef]

24. Ota, H.; Yarnykh, V.L.; Ferguson, M.S.; Underhill, H.R.; Demarco, J.K.; Zhu, D.C.; Oikawa, M.; Dong, L.; Zhao, X.; Collar, A.; et al. Carotid intraplaque hemorrhage imaging at 3.0-T MR imaging: Comparison of the diagnostic performance of three T1-weighted sequences. Radiology 2010, 254, 551-563. [CrossRef]

25. Li, D.; Zhao, H.; Chen, X.; Chen, S.; Qiao, H.; He, L.; Li, R.; Xu, J.; Yuan, C.; Zhao, X. Identification of intraplaque haemorrhage in carotid artery by simultaneous non-contrast angiography and intraPlaque haemorrhage (SNAP) imaging: A magnetic resonance vessel wall imaging study. Eur. Radiol. 2018, 28, 1681-1686. [CrossRef] [PubMed]

26. Li, D.; Qiao, H.; Han, Y.; Han, H.; Yang, D.; Cao, J.; Xu, H.; Wang, T.; Wang, Y.; Shen, J.; et al. Histological validation of simultaneous non-contrast angiography and intraplaque hemorrhage imaging (SNAP) for characterizing carotid intraplaque hemorrhage. Eur. Radiol. 2020, 31, 3106-3115. [CrossRef]

27. Tsukahara, T.; Minematsu, K. Overview of spontaneous cervicocephalic arterial dissection in Japan. In Surgical Management of Cerebrovascular Disease; Springer: Berlin/Heidelberg, Germany, 2010; pp. 35-40.

28. Wang, Y.; Lou, X.; Li, Y.; Sui, B.; Sun, S.; Li, C.; Jiang, P.; Siddiqui, A.; Yang, X. Imaging investigation of intracranial arterial dissecting aneurysms by using $3 \mathrm{~T}$ high-resolution MRI and DSA: From the interventional neuroradiologists' view. Acta Neurochir. 2014, 156, 515-525. [CrossRef] [PubMed]

29. Gao, P.; Yang, L.; Wang, G.; Guo, L.; Liu, X.; Zhao, B. Symptomatic unruptured isolated middle cerebral artery dissection: Clinical and magnetic resonance imaging features. Clin. Neuroradiol. 2016, 26, 81-91. [CrossRef] [PubMed]

30. Sakurai, K.; Miura, T.; Sagisaka, T.; Hattori, M.; Matsukawa, N.; Mase, M.; Kasai, H.; Arai, N.; Kawai, T.; Shimohira, M. Evaluation of luminal and vessel wall abnormalities in subacute and other stages of intracranial vertebrobasilar artery dissections using the volume isotropic turbo-spin-echo acquisition (VISTA) sequence: A preliminary study. J. Neuroradiol. 2013, 40, 19-28. [CrossRef] [PubMed]

31. Pfefferkorn, T.; Saam, T.; Rominger, A.; Habs, M.; Gerdes, L.-A.; Schmidt, C.; Cyran, C.; Straube, A.; Linn, J.; Nikolaou, K. Vessel wall inflammation in spontaneous cervical artery dissection: A prospective, observational positron emission tomography, computed tomography, and magnetic resonance imaging study. Stroke 2011, 42, 1563-1568. [CrossRef] [PubMed]

32. Sakamoto, Y.; Okubo, S.; Nito, C.; Suda, S.; Matsumoto, N.; Abe, A.; Aoki, J.; Shimoyama, T.; Muraga, K.; Kanamaru, T.; et al. The Prevalence of and Factors Related to Vascular Hyperintensity on T1-Weighted Imaging in Acute Ischemic Stroke. Cerebrovasc. Dis. 2017, 44, 203-209. [CrossRef] 